\title{
Design and Analysis of Optimal Random Access Policies in Cognitive Radio Networks
}

\author{
Gang Uk Hwang, Member, IEEE, and Sumit Roy, Fellow, IEEE
}

\begin{abstract}
In this paper, we consider a time slotted cognitive radio network where multiple secondary users (SUs) contend to access wireless channels. We propose and analyze a channel access policy where each secondary user stochastically determines whether to access a wireless channel (or not) based on a given access probability. In our analysis we consider two extreme cases - where all SUs a) possess full information and b) have no information regarding idle channels. We propose to obtain the access probabilities that minimize the steady-state queue length tail probabilities in the two extreme cases based on Effective Bandwidth theory. Our analysis provides an insight on how to design optimal channel access policies in the two extreme cases. We also show how the optimal channel access policies of two extreme cases are related with each other. Some numerical examples are provided to validate our analysis and investigate the performance behaviors of the optimal channel access policies.
\end{abstract}

Index Terms-Cognitive radio networks, channel access policy, effective bandwidth theory, performance analysis, tail probability.

\section{INTRODUCTION}

$\mathbf{S}$ INCE radio spectrum is a scarce resource in wireless networks, spectral efficiency is one of the important performance metrics for wireless communications. Recent actual measurements have shown that most of the allocated spectrum is under-utilized [1]-[3]. On the other hand, the demands for radio spectrum to support new wireless services have been increasing. To improve inefficient use of allocated spectrum, the concept of cognitive radio has been introduced. Such networks consist of two types of users - licensed and unlicensed. The former have absolute priority to occupy the assigned radio spectrum, while unlicensed users are potentially allowed to use the spectrum when it is not 'occupied' by the licensed users. We adopt the conventional terminology within cognitive radio community: licensed users are the primary users (PUs) and unlicensed users are the secondary users (SUs).

In this paper, we consider a time slotted wireless network with total of $N$ channels. To discover the channels unoccupied by PUs (the 'idle' channels), SUs must first sense channel state (either individually or collectively) at the beginning of each slot. After obtaining such channel state information, each SU then selects (one or more) idle channels for communications

Paper approved by A. MacKenzie, the Editor for Game Theory and Cognitive Networking of the IEEE Communications Society. Manuscript received November 15, 2010; revised May 25, 2011 and August 10, 2011.

G. U. Hwang is with the Department of Mathematical Sciences, Korea Advanced Institute of Science and Technology (KAIST), Korea (e-mail: guhwang@kaist.edu).

S. Roy is with the Department of Electrical Engineering, University of Washington, Seattle (e-mail: roy@ee.washington.edu).

Digital Object Identifier 10.1109/TCOMM.2011.112311.100702 according to a pre-determined channel access policy. Hence, a suitable choice of channel sensing and access policies is fundamental to improving spectral efficiency and network performance.

The rapidly growing literature on cognitive networks already contains several works on channel sensing and channel access (MAC) policies that directly impact the performance of such networks. A brief summary of relevant works is provided in Section II. At this time, there have been relatively few attempts at true cross-layer formulations that connect MAC layer performance to physical layer sensing [4], [5]. In this work, we focus on developing queuing models for dynamic cognitive MAC protocols - an area where the prior art is relatively sparse. Since loss (due to buffer overflows) is an important QoS metric for multimedia flows, we use buffer overflow (loss) probability as the driving optimality criterion. While the present formulation is not cross-layer, such models are a necessary first step towards future work that incorporates the impact of (imperfect) channel sensing.

We consider a time slotted network with $N$ wireless channels and $M$ SUs. We focus on a channel access policy for SUs that we analyze under two extreme cases - a) where all SUs have full channel state information (i.e., which channels are idle and which are busy) and b) where all SUs do not have any information on idle channels. SUs may obtain reliable channel state information via a suitable channel sensing policy such as the negotiation-based sensing in [4]* or a centralized database as mandated by the FCC is provided to SUs. In our work, each SU determines whether to access an idle channel at each slot based on the common access probability (AP). If two or more SUs randomly select the same idle channel, it leads to a collision. Clearly, a policy that adapts the AP to the knowledge of the idle channels so as to mitigate collisions, is desirable. On the other hand, when SUs do not have any information on idle channels, a fixed AP is used irrespective of the number of idle channels. For both scenarios, it is desirable to obtain the optimal values of APs to optimize queueing performance.

In this paper, we use the tail probability of the queue length at any SU as our performance metric, adopting the fact that it is widely used to estimate the packet overflow probability and delay. The tail probability has been estimated using effective bandwidth theory initially for wireline packet networks [6]. Recently, effective bandwidth theory has been used to analyze MAC queueing performance of wireless networks e.g. [7]-[9]. In our analysis we derive the effective bandwidth function (EBF) of the packet service process for a given choice of

*In [4] they assume $M \geq N$, but even when $M<N$, SUs may obtain full information on idle channels by slightly modifying the negotiation-based sensing policy. 
APs. We subsequently analyze the characteristics of the EBF obtained and derive the optimal values of APs in the two extreme cases listed above. Our analysis provides explicit expressions for the optimal AP values in the channel access schemes and shows a direct relation between the optimal $\mathrm{AP}$ values and network performance. We also show how the optimal channel access policies of two extreme cases are related with each other.

The organization of this paper is as follows. In Section II we provide a brief summary on related works. In Section III we consider a cognitive radio network with homogeneous wireless channels and provide our mathematical model for the cognitive radio network. We also explain our channel access policy in detail in Section III. In Section IV we analyze queueing performance with the help of effective bandwidth theory and prove our main theorems on the optimal channel access policies for two extreme cases. In Section VI we extend the homogeneous channel model to the heterogeneous channel model and analyze how to obtain the optimal APs for the heterogeneous channel model. In Section VII we provide some numerical examples to validate our analysis and to investigate the queueing performance. In Section VIII we give our conclusions.

\section{LiterATURE REVIEW}

Estimating channel state information in cognitive radio networks is fundamentally related to (physical layer) sensing modality employed. The key questions are related to a) the sensing duration per channel (that impacts the accuracy of determining channel state - busy or idle) and b) the order of sensing of the channels (that impacts the mean duration to identifying a busy channel) [10], [11]. Hence, optimizing of channel sensing has been dealt with in several works, notably [12]-[14]. In [15] they propose an optimal stopping rule for channel sensing by considering hardware constraints. [10] jointly investigates channel sensing duration and search order optimization problems. In [5] a myopic sensing policy based on a partially observable Markov decision process (POMDP) framework is proposed. [4] suggests a new MAC protocol with two phases - reporting (wherein all SUs sense the channel state cooperatively) followed by negotiation-based channel access.

Channel access protocols for cognitive networks are classified into three categories in the survey by [11]- random access, time slotted and hybrid. Among the many channel access policies proposed include Dynamic open spectrum sharing (DOSS) MAC [16], Hardware constraint MAC (HC-MaC) [15], Cognitive MAC (C-MAC) [17], POMDP based MAC [18], Reporting and Negotiation based MAC [4]. Unfortunately, few of these works focus on queueing performance analysis for a cognitive (SU) user. An $M / G / 1$ queueing model is proposed to analyze the multiplexing gain and delay performance of a cognitive radio network in [19]. In [20], analytical models are developed for two dynamic spectrum access protocols, to investigate their latency performances. In [4], an $M / G^{Y} / 1$ queueing system is used to analyze their negotiation-based MAC protocol. In [21] a queueing analytic framework is developed to study the MAC performance with opportunistic spectrum scheduling. The queueing performance of the myopic sensing policy [5] is analyzed by using a large deviation approach in [22]. In [23] the queueing delay of a cognitive radio network is analyzed where each SU that identifies an idle channel contends for it with a fixed probability. The cognitive radio network considered in [23] is identical to one of our cases where SUs do not have any channel information. They obtained the optimal contention probability that minimizes the mean queue lengths.

\section{Mathematical Modeling}

\section{A. Wireless Channel Occupancy Model}

We consider a slotted, cognitive radio network with $N$ wireless channels, where time is indexed by $t(t=0,1,2, \cdots)$. For each channel, the channel occupancy by primary users (PUs) is modeled by a two state Markov chain with state space $\{0,1\}$, where $0(1)$ denotes a channel that is occupied (free). We assume that state transitions occur at slot boundaries and the transition probability matrix of the Markov chain is given by

$$
\mathbf{Q}:=\left(\begin{array}{cc}
1-p & p \\
q & 1-q
\end{array}\right)
$$

where $p$ is the transition probability from state 0 to state 1 and $q$ is the transition probability from state 1 to state 0 . Let $\boldsymbol{\pi}=\left(\pi_{0}, \pi_{1}\right)$ be the stationary probability vector of the matrix $\mathbf{Q}$, i.e., $\boldsymbol{\pi} \mathbf{Q}=\boldsymbol{\pi}, \pi_{0}+\pi_{1}=1$. It is then easy to show that

$$
\pi_{0}=\frac{q}{p+q}, \pi_{1}=\frac{p}{p+q} .
$$

Let $N(t)$ be the number of idle wireless channels at time $t$. It follows that $N(t)$ is a Discrete Time Markov Chain (DTMC) with state space $\{0,1, \cdots, N\}$. The transition probability matrix of $N(t)$ is denoted by $\mathbf{R}=\left(R_{k l}\right)$ where $R_{k l}$ denotes the $(k, l)$-th element of the matrix $\mathbf{R}$ and is given by

$$
\begin{aligned}
R_{k l}=\sum_{i=\max (0, k+l-N)}^{\min (k . l)}\left(\begin{array}{c}
k \\
i
\end{array}\right)(1-q)^{i} q^{k-i} \\
\quad \times\left(\begin{array}{c}
N-k \\
l-i
\end{array}\right) p^{l-i}(1-p)^{N-k-l+i} .
\end{aligned}
$$

\section{B. Wireless Channel Access Policy}

There are $M(\geq 2)$ secondary users (SUs) in the network.

Case 1: SUs have perfect knowledge of the state of all $N$ channels at each slot, i.e. the specific idle (occupied) channels are known to all SUs.

An SU stochastically determines whether to access a channel at each slot by using the access probability (AP), independently of all other SUs. For $N(t)=k$, each SU accesses an idle channel with probability $a_{k}$ and does not access an idle channel with probability $1-a_{k}$. The set of SUs that decides to access channels is termed the 'active' set. Each active SU then randomly (i.e., uniformly) selects one among the known set of idle channels and transmits one packet during a slot. Whenever two or more active SUs select the same idle channel, their transmissions result in a collision and are assumed lost. All collided packets are prioritized for retransmission by SUs in the next available slots. Only when an idle channel is selected by only one active SU is the transmission successful, i.e., we implicitly assume that there is no other sources of loss (such 
as link loss). Clearly, the values of APs $\left\{a_{1}, a_{2}, \cdots, a_{N}\right\}$ significantly affects the queueing performances of SUs. When the number of idle channels $(N(t))$ is small (large), fewer (greater) active SUs is desirable. So the APs $\left\{a_{1}, a_{2}, \cdots, a_{N}\right\}$ need to be optimized for the optimal queueing performances of SUs.

Case 2: SUs have no channel state information.

Here, all SUs cannot adapt the AP according to the number of idle channels. Instead, they use a common value for the $\mathrm{AP}$, say $a$, to determine whether they are active or not at each slot, independently of all other SUs. Then, when an SU becomes active, it randomly selects one of the $N$ channels. The active SU then checks at the beginning of the slot whether the selected channel is busy at the slot, i.e., a PU occupies the selected channel. If the selected channel is busy, then the active SU does not transmit its packet at the slot to avoid interfering with the PU's transmission. Otherwise, the active SU transmits its packet through the selected channel. Obviously, there is the possibility of packet collisions when two or more active SUs select the same idle channel. So, as in the previous case, the AP $a$ need to be optimized. The benefit of this policy is that each active SU senses only one (randomly chosen) channel at each slot and does not need any information about the other channels, reducing the sensing overhead.

The objective of this work is to determine the optimal values of APs $\left\{a_{1}, a_{2}, \cdots, a_{N}\right\}$ or $a$ that optimize the queueing performance of each SU. Adopting the fact that the tail probability of the queue length is widely used to estimate the packet overflow probability and delay [9], we use it as the performance metric in this paper. To obtain the optimal values of APs, we use the effective bandwidth theory which is briefly explained in the next section.

\section{Queueing Performance And EfFective BANDWIDTH THEORY}

\section{A. Queueing Model and Effective Bandwidth Theory}

We assume that each SU has a buffer to accommodate its packets at the MAC layer. We select an arbitrary SU as the reference and consider the packet service process of the tagged SU at the MAC layer. Let $c_{N(t)}(t)$ be the packet service process related with the service capacity for the tagged SU. That is, assuming that the tagged SU has a packet to transmit and $N(t)=k$ at slot $t, c_{k}(t)$ is then defined as follows: $c_{k}(t)=1$ if the transmission by the tagged $\mathrm{SU}$ is successful at slot $t$, and 0 otherwise.

Let $L(t)(t=0,1, \ldots)$ denote the queue length (i.e., the number of packets in the queue) of the tagged SU at slot $t$, and $a(t)(t=0,1, \ldots)$ the number of new packets arriving at the tagged SU at slot $t$. Since $c_{N(t)}(t)$ is the packet service process related with the service capacity of the tagged $\mathrm{SU}$, the queueing process $\{L(t)\}$ evolves according to the following recursion [6], [24]:

$L(0)=0, L(t+1)=\max \left\{0, L(t)+a(t)-c_{N(t)}(t)\right\}, t \geq 0$.

To analyze the queueing process $\{L(t)\}$, we use effective bandwidth theory which has been widely used for the MAC performance analysis in wireless networks, e.g., [7]-[9]. In effective bandwidth theory, we need to compute two effective

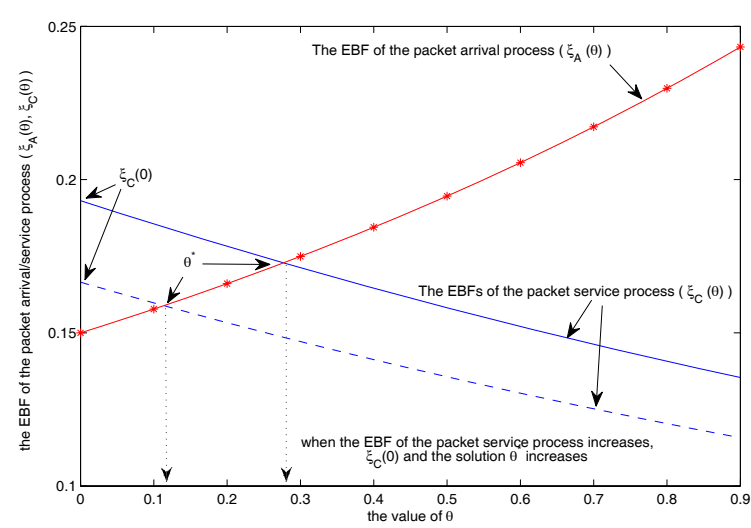

Fig. 1. The increase of the effective bandwidth function vs. $\xi_{C}(0)$ and $\theta^{*}$.

bandwidth functions (EBFs): one for the packet service process $c_{N(t)}(t)$ and the other for the packet arrival process $a(t)$. The EBF for the packet service process is sometimes called the effective capacity, e.g., [7].

We start with deriving the EBF for the packet service process for the tagged SU. Let $C(t)(t=0,1, \ldots)$ denote the cumulative service process during the interval $[0, t)$, i.e., $C(t)=\sum_{s=0}^{t-1} c_{N(s)}(s)$. The Gärtner-Ellis limit $\Lambda_{C}(\theta)$ of the cumulative service process $C(t)$ is defined by $\Lambda_{C}(\theta)=$ $\lim _{t \rightarrow \infty} \frac{1}{t} \log (\mathrm{E}[\exp (\theta C(t))])$. Then the EBF of the packet service process is defined by [6], [7]

$$
\xi_{C}(\theta):=-\frac{\Lambda_{C}(-\theta)}{\theta} .
$$

The EBF of the packet service process, $\xi_{C}(\theta)$ captures the stochastic properties of the packet service process $c_{N(t)}(t)$. For instance, when $\theta \rightarrow 0$, it converges to the average service rate. In contrast, when $\theta \rightarrow \infty$, it converges to the minimum service rate [6], [9]. A typical plot of the EBF of the packet service process is shown in Fig. 1. In the figure, two decreasing functions of $\theta$, denoted by $\xi_{C}(\theta)$, are the EBFs of the packet service processes. Fig. 1 will be used to explain how the optimal values of APs are obtained in Section IV-B.

We next derive the EBF of the packet arrival process at the tagged SU. Let $A(t)(t=0,1, \ldots)$ denote the cumulative arrival process during the interval $[0, t)$, i.e., $A(t)=\sum_{n=0}^{t-1} a(n)$. We define the EBF of the arrival process, $\xi_{A}(\theta)$, by [6], [7]

$$
\xi_{A}(\theta):=\frac{\Lambda_{A}(\theta)}{\theta},
$$

where

$$
\Lambda_{A}(\theta)=\lim _{t \rightarrow \infty} \frac{1}{t} \log (\mathrm{E}[\exp (\theta A(t))]) .
$$

Similar to $\xi_{C}(\theta)$, the EBF of the packet arrival process captures the stochastic properties of the packet arrival process. For instance, when $\theta \rightarrow 0$, it converges to the average arrival rate; for $\theta \rightarrow \infty$, it converges to the peak arrival rate. A typical plot of the EBF of the packet arrival process is also shown in Fig. 1. That is, the increasing function of $\theta$, denoted by $\xi_{A}(\theta)$, is the EBF of the packet arrival process. For more details on the properties of the EBFs, interested readers may refer to [6], [9].

With the two $\operatorname{EBFs} \xi_{C}(\theta)$ and $\xi_{A}(\theta)$, we determine the queueing performance of the tagged SU as follows. Let $L(\infty)$ 
denote the queue length from (2) in the steady state. It is known that for stable queues, the tail probability $\mathrm{P}(L(\infty)>$ $x)$ in the steady state is approximately given by [6], [7], [25]

$$
\mathrm{P}(L(\infty)>x) \approx \mathrm{P}(L(\infty)>0) \exp \left(-\theta^{*} x\right),
$$

where $\theta^{*}$ is the unique real solution to the equation

$$
\xi_{A}(\theta)=\xi_{C}(\theta) .
$$

In addition, we have

$$
\mathrm{P}(L(\infty)>0)=\frac{\xi_{A}(0)}{\xi_{C}(0)} .
$$

It has been known that the approximation (3) provides a good prediction of the buffer overflow probability for a wide range of queueing systems [9]. In this paper, the optimization in queueing performance means the minimization of $\mathrm{P}(L(\infty)>$ $x)$, or equivalently, the maximization of $\xi_{C}(0)$ and $\theta^{*}$ due to (4) and (5).

For later use, we provide an explicit expression for the EBF of the packet arrival process, $\xi_{A}(\theta)$. For simplicity ${ }^{\dagger}$, we assume that the packet arrival process at the tagged $\mathrm{SU}$ is according to a Poisson process with arrival rate $\lambda$ (packets/slot). In this case, the EBF of the packet arrival process is given by [6]

$$
\xi_{A}(\theta)=\frac{\lambda\left(e^{\theta}-1\right)}{\theta}
$$

\section{B. The Optimization Problem in Queueing Performance}

To explain how we obtain the optimal values of APs that maximize $\xi_{C}(0)$ and $\theta^{*}$, we consider two service policies in a wireless network and assume that their corresponding EBFs are given as in Fig. 1 (shown with solid and dashed line, respectively). Obviously from (3),(4), (5), and Fig. 1, the EBF with solid line results in larger values of $\xi_{C}(0)$ and $\theta^{*}$ than the EBF with dashed line. This implies that the service policy corresponding to the EBF with solid line outperforms the service policy corresponding to the EBF with dashed line. Based on this observation, if we can design a service policy that results in the largest $\mathrm{EBF}$ for all $\theta(\geq 0)$, then the resulting $\xi_{C}(0)$ and $\theta^{*}$ are the optimum values.

Recall that our objective is to determine the optimal APs $\left\{a_{1}^{*}, a_{2}^{*}, \cdots, a_{N}^{*}\right\}$ and $a^{*}$ that maximize $\xi_{C}(0)$ and $\theta^{*}$ for the two cases. Hence, from the discussion above, we see that this is equivalent to finding the $\mathrm{AP}$ values $\left\{a_{1}^{*}, a_{2}^{*}, \cdots, a_{N}^{*}\right\}$ and $a^{*}$ that maximize the EBF of the packet service process. Hence, our next step is to explicitly obtain $\xi_{C}(\theta)$ for our channel access policy, as is derived in the next section.

\section{Analysis for The Optimal Design}

In this section, we analyze the characteristics of the EBF of the packet service process and show how to obtain the optimal values of APs. To this end, we assume that all untagged SUs have packets to transmit, and participate in the channel access process to determine whether they are active or not based on the AP.

\footnotetext{
${ }^{\dagger}$ Even though we assume the Poisson arrivals in this study, our analytic framework can accommodate more general arrival processes.
}

To obtain the EBF of the packet service process, we first note that when the packet service process $\left\{c_{N(t)}(t)\right\}$ is a Markov modulated process, with the help of the matrix theory, we can obtain a more explicit expression for $\xi_{C}(\theta)$ as follows. Let $\phi(\theta)$ be the diagonal matrix with diagonal elements $\left\{\phi_{0}(\theta), \phi_{1}(\theta), \ldots, \phi_{N}(\theta)\right\}$ where $\phi_{k}(\theta)$ are defined by

$$
\phi_{k}(\theta):=\mathrm{E}\left[e^{\theta c_{N(t)}(t)} \mid N(t)=k\right], \quad 0 \leq k \leq N .
$$

Then, it can be shown that the EBF of the packet service process, $\xi_{C}(\theta)$, is given by

$$
\xi_{C}(\theta)=-\frac{\log \delta_{C}(-\theta)}{\theta},
$$

where $\delta_{C}(\theta)$ is the Perron-Frobenius (PF) eigenvalue of the matrix

$$
C(\theta)=\phi(\theta) \mathbf{R} .
$$

For the proof, refer to [6], [24].

We next derive the diagonal elements $\phi_{k}(\theta)$ in (6) and show how to obtain the optimal values of APs for the two cases of interest.

\section{A. Case 1: Full Channel State Information}

We assume that all SUs have perfect information of channel state, i.e., know which channels are idle and which are busy. We derive an expression for $\phi_{k}(\theta)$ in (6), which we denote by $\phi_{k}^{\text {full }}(\theta)$ for this case.

When $k=0$, it is obvious that $\phi_{0}^{\text {full }}(\theta)=1$ because $c_{N(t)}(t)=0$ for $N(t)=0$. For $1 \leq k \leq N, \phi_{k}^{\text {full }}(\theta)$ is given as follows:

$$
\begin{aligned}
& \phi_{k}^{f u l l}(\theta):=\mathrm{E}\left[e^{\theta c_{N(t)}(t)} \mid N(t)=k\right] \\
& =a_{k} \sum_{i=0}^{M-1}\left(\begin{array}{c}
M-1 \\
i
\end{array}\right) a_{k}^{i}\left(1-a_{k}\right)^{M-i-1}\left(\frac{k-1}{k}\right)^{i} e^{\theta} \\
& +1-a_{k} \sum_{i=0}^{M-1}\left(\begin{array}{c}
M-1 \\
i
\end{array}\right) a_{k}^{i}\left(1-a_{k}\right)^{M-i-1}\left(\frac{k-1}{k}\right)^{i} \\
& =a_{k}\left(a_{k} \frac{k-1}{k}+1-a_{k}\right)^{M-1} e^{\theta} \\
& \quad+1-a_{k}\left(a_{k} \frac{k-1}{k}+1-a_{k}\right)^{M-1} \\
& =1+a_{k}\left(a_{k} \frac{k-1}{k}+1-a_{k}\right)^{M-1}\left(e^{\theta}-1\right) \\
& =1+a_{k}\left(1-a_{k} \frac{1}{k}\right)^{M-1}\left(e^{\theta}-1\right) .
\end{aligned}
$$

In the RHS of the second equation, $a_{k}$ is the probability that the tagged SU becomes active, $\left(\begin{array}{c}M-1 \\ i\end{array}\right) a_{k}^{i}\left(1-a_{k}\right)^{M-i-1}$ is the probability that there are $i$ untagged active SUs, and $\left(\frac{k-1}{k}\right)^{i}$ is the probability that all untagged active SUs do not select the same idle channel that the tagged SU selects.

Lemma 1: For $1 \leq k \leq N, \phi_{k}^{\text {full }}(-\theta)$ is minimized for each $\theta(>0)$ when $a_{k}=\min \left(\frac{k}{M}, 1\right)$.

Proof: For each $k$, define $f_{k}(x)$ as

$$
f_{k}(x):=x\left(1-x \frac{1}{k}\right)^{M-1}, 0 \leq x \leq 1, M \geq 2 .
$$


By differentiating $f_{k}(x)$, we get

$$
f_{k}^{\prime}(x)=\left(1-x \frac{1}{k}\right)^{M-2}\left(1-x \frac{M}{k}\right) .
$$

By verifying $f_{k}^{\prime}(x) \geq 0$, we see that $f_{k}(x)$ strictly increases for $0 \leq x<\min \left(\frac{k}{M}, 1\right)$ and strictly decreases for $\min \left(\frac{k}{M}, 1\right)<x \leq 1$. Hence, $f_{k}(x)$ is maximized when $x=\min \left(\frac{k}{M}, 1\right)$. We next observe that

$$
\phi_{k}^{f u l l}(-\theta)=1+f_{k}\left(a_{k}\right)\left[e^{-\theta}-1\right], \theta>0 .
$$

Since $e^{-\theta}-1<0$ for $\theta>0$, the maximization of $f_{k}(x)$ results in the minimization of $\phi_{k}^{f u l l}(-\theta)$. Accordingly $\phi_{k}^{\text {full }}(-\theta)$ is minimized when $a_{k}=\min \left(\frac{k}{M}, 1\right)$ for each $\theta>0$.

Recall that the EBF of the packet service process is related to the PF eigenvalue of the matrix $C^{f u l l}(-\theta):=\phi^{f u l l}(-\theta) \mathbf{R}$, which will be used in our analysis with the help of the following result proved in [26].

Proposition 1: If $\mathbf{A}$ is an irreducible matrix, and $\mathbf{A} \geq \mathbf{B} \geq$ $\mathbf{0}, \mathbf{A} \neq \mathbf{B}$, then the PF eigenvalue of $\mathbf{A}$ is larger than that of B.

Since $\mathbf{R}$ is irreducible by its definition and all elements $\phi_{k}^{f u l l}(-\theta)$ of the diagonal matrix $\phi^{\text {full }}(-\theta)$ are positive for $\theta>0, C^{\text {full }}(-\theta)$ is also irreducible for $\theta>0$. With the help of Proposition 1 we have our first main theorem.

Theorem 1: The optimal values of APs $\left\{a_{1}^{*}, a_{2}^{*}, \cdots, a_{N}^{*}\right\}$ satisfy

$$
a_{k}^{*}=\min \left(\frac{k}{M}, 1\right), 1 \leq k \leq N .
$$

Proof: For the proof, we will omit the superscript full for simplicity. For APs $\left\{a_{1}, a_{2}, \cdots, a_{N}\right\}$ and $N(t)=k$, the service capacity for the tagged $\mathrm{SU}$ is denoted by $c_{k}\left(t ; a_{k}\right)$ (which corresponds to $c_{k}(t)$ in Section IV). Let $\delta_{C}\left(\theta ; a_{1}, a_{2}, \cdots, a_{N}\right)$ be the PF eigenvalue of

$$
\boldsymbol{C}(\theta):=\phi\left(\theta ; a_{1}, a_{2}, \cdots, a_{N}\right) \mathbf{R}
$$

where $\phi\left(\theta ; a_{1}, a_{2}, \cdots, a_{N}\right)$ is a diagonal matrix whose $k$-th element is denoted by $\phi_{k}\left(\theta ; a_{k}\right)$ and is defined by

$$
\phi_{k}\left(\theta ; a_{k}\right):=\mathrm{E}\left[e^{\theta c_{k}\left(t ; a_{k}\right)} \mid N(t)=k\right] .
$$

Then the EBF of the packet service process when we use $\left\{a_{1}, a_{2}, \cdots, a_{N}\right\}$ is given by

$$
\xi_{C}\left(\theta ; a_{1}, a_{2}, \cdots, a_{N}\right)=-\frac{\log \delta_{C}\left(-\theta ; a_{1}, a_{2}, \cdots, a_{N}\right)}{\theta}
$$

where $\delta_{C}\left(-\theta ; a_{1}, a_{2}, \cdots, a_{N}\right)$ is the PF eigenvalue of the matrix $\boldsymbol{C}(-\theta)$.

We next consider the EBF of the packet service process when we use $\left\{a_{1}^{*}, a_{2}, \cdots, a_{N}\right\}$, that is, the $\mathrm{AP} a_{1}\left(\neq a_{1}^{*}\right)$ for $N(t)=1$ is replaced by $a_{1}^{*}$. Then the resulting EBF is given by

$$
\xi_{C}\left(\theta ; a_{1}^{*}, a_{2}, \cdots, a_{N}\right)=-\frac{\log \delta_{C}\left(-\theta ; a_{1}^{*}, a_{2}, \cdots, a_{N}\right)}{\theta} .
$$

In what follows we show $\xi_{C}\left(\theta ; a_{1}^{*}, a_{2}, \cdots, a_{N}\right)>$ $\xi_{C}\left(\theta ; a_{1}, a_{2}, \cdots, a_{N}\right)$ for each $\theta>0$. To this end, we first observe from Lemma 1 that

$$
\phi_{1}\left(-\theta ; a_{1}\right)>\phi_{1}\left(-\theta ; a_{1}^{*}\right)
$$

and the other elements $\phi_{k}\left(\theta ; a_{k}\right), 0 \leq k \leq N, k \neq 1$, remain invariant. By Proposition 1 we know that

$$
\delta_{C}\left(-\theta ; a_{1}, a_{2}, \cdots, a_{N}\right)>\delta_{C}\left(-\theta ; a_{1}^{*}, a_{2}, \cdots, a_{N}\right)
$$

and accordingly, by taking logarithms and dividing $-\theta(<0)$ on both sides of the above equation we get

$$
\xi_{C}\left(\theta ; a_{1}, a_{2}, \cdots, a_{N}\right)<\xi_{C}\left(\theta ; a_{1}^{*}, a_{2}, \cdots, a_{N}\right) .
$$

We next consecutively change the values of $a_{2}, \cdots, a_{N}$ to $a_{2}^{*}, \cdots, a_{N}^{*}$ and use the above arguments to show that

$$
\xi_{C}\left(\theta ; a_{1}, a_{2}, \cdots, a_{N}\right)<\xi_{C}\left(\theta ; a_{1}^{*}, a_{2}^{*}, \cdots, a_{N}^{*}\right) .
$$

This implies that, when we use the values $\left\{a_{1}^{*}, a_{2}^{*}, \cdots, a_{N}^{*}\right\}$ of APs, the resulting EBF of the packet service process is maximized for each $\theta(>0)$ and hence queueing performance is optimized.

From Theorem 1 we see that the optimal APs are proportional to the number of idle channels and inversely proportional to the number of SUs. The intuitive explanation on Theorem 1 is as follows. The optimal AP increases in the number of idle channels. This implies that, when there are more idle channels, the network allows more SUs to access idle channels and this results in more successful packet transmissions. On the other hand, when there are very few idle channels, the network allows only few SUs to access idle channels and hence it can alleviate packet collisions.

In addition, the optimal AP decreases in the number of SUs. That is, the more SUs in the network the less AP value for each SU. By doing this, the network becomes less crowded from the channel's viewpoint even when the number of SUs is large, and accordingly the network gets its optimal performance.

\section{B. Case 2: No Channel State Information}

In this case, all SUs use the unified AP, $a$, to determine whether they are active or not to access channels. To get the optimal value $a^{*}$ of AP in this case, we start with $\phi_{k}(\theta)$ defined in (6) as in Subsection V-A, but again by the abuse of notation we use $\phi_{k}^{n o}(\theta)$ instead of $\phi_{k}(\theta)$. When $k=0$, we obviously have $\phi_{0}^{n o}(\theta)=1$. For $1 \leq k \leq N, \phi_{k}^{n o}(\theta)$ is given as follows:

$$
\begin{aligned}
\phi_{k}^{n o}(\theta) & :=\mathrm{E}\left[e^{\theta c_{N(t)}(t)} \mid N(t)=k\right] \\
= & a \frac{k}{N} \sum_{i=0}^{M-1}\left(\begin{array}{c}
M-1 \\
i
\end{array}\right) a^{i}(1-a)^{M-i-1}\left(\frac{N-1}{N}\right)^{i} e^{\theta} \\
& +1-a \frac{k}{N} \sum_{i=0}^{M-1}\left(\begin{array}{c}
M-1 \\
i
\end{array}\right) a^{i}(1-a)^{M-i-1}\left(\frac{N-1}{N}\right)^{i} \\
= & a \frac{k}{N}\left(a \frac{N-1}{N}+1-a\right)^{M-1} e^{\theta} \\
& +1-a \frac{k}{N}\left(a \frac{N-1}{N}+1-a\right)^{M-1} \\
= & 1+a \frac{k}{N}\left(a \frac{N-1}{N}+1-a\right)^{M-1}\left(e^{\theta}-1\right) \\
= & 1+a \frac{k}{N}\left(1-a \frac{1}{N}\right)^{M-1}\left(e^{\theta}-1\right) .
\end{aligned}
$$

In the RHS of the second equation, $a$ is the probability that the tagged SU becomes active, $\frac{k}{N}$ is the probability that the 
tagged SU selects an idle channel, $\left(\begin{array}{c}M-1 \\ i\end{array}\right) a^{i}(1-a)^{M-i-1}$ is the probability that there are $i$ untagged active SUs, and $\left(\frac{N-1}{N}\right)^{i}$ is the probability that all untagged active SUs do not select the same channel that the tagged SU selects.

Using $\phi_{k}^{n o}(\theta)$ in (10) and the same argument provided in Subsection V-A, we prove the following theorem for the optimal value $a^{*}$ of the AP in this case.

Theorem 2: The optimal AP value satisfies $a^{*}=$ $\min \left(\frac{N}{M}, 1\right)$.

A comparison of Theorem 2 with Theorem 1 reveals the following interesting observation. In the case of no channel state information, the optimal queueing performance can be achieved when the tagged SU acts as if all channels were idle. Further, we prove the following theorem which shows the equivalence between the optimal channel access policies for the two extreme cases, when the number of channels is less than or equal to the number of SUs, i.e., $N \leq M$.

Theorem 3: For $N \leq M$, the optimal channel access policy for the case of no channel state information is equivalent to the optimal channel access policy for the case of full channel state information from the queueing performance point of view.

Proof: To prove our theorem, we consider $\phi_{k}^{\text {full }}(\theta)$ in (9) and $\phi_{k}^{n o}(\theta)$ in (10). When $k=0$,

$$
\phi_{0}^{\text {full }}(\theta)=1=\phi_{0}^{n o}(\theta) .
$$

When $1 \leq k \leq N$, since $N \leq M$, the optimal APs in the case of full information are given by $a_{k}^{*}=\frac{k}{M}$. By substituting $a_{k}^{*}$ for $a_{k}$ in $\phi_{k}^{f u l l}(\theta)$, we get from (9) that

$$
\left.\phi_{k}^{\text {full }}(\theta)\right|_{a_{k}=a_{k}^{*}}=1+\frac{k}{M}\left(1-\frac{1}{M}\right)^{M-1}\left(e^{\theta}-1\right) .
$$

Similarly, since the optimal AP in the case of no information is given by $a^{*}=\frac{N}{M}$, we get from (10) that

$$
\left.\phi_{k}^{n o}(\theta)\right|_{a=a^{*}}=1+\frac{k}{M}\left(1-\frac{1}{M}\right)^{M-1}\left(e^{\theta}-1\right) .
$$

Therefore

$$
\left.\phi_{k}^{f u l l}(\theta)\right|_{a_{k}=a_{k}^{*}}=\left.\phi_{k}^{n o}(\theta)\right|_{a=a^{*}} .
$$

The respective elements of the matrix $\boldsymbol{C}(\theta)$ defined in (8) for both cases of full and no information are identical, and accordingly the resulting PF eigenvalues in (7) for both cases of full and no information are the same. That is, the EBFs of the packet service processes are identical for both cases. This shows the equivalence between the optimal channel access policies of both cases of full and no channel state information.

Hence, if we combine Theorem 2 and Theorem 3 together, we conclude that the optimal queueing performance can be still achieved if all SUs acts optimistically (i.e., as if all channels were idle), even when they have no information on channel state and $N \leq M$.

\section{Vi. Generalization For Heterogeneous Channels}

Up to now we assume that all wireless channels are homogeneous with the same state transition probability matrix $\mathbf{Q}$ given in Section III-A. In this section, we consider the heterogeneous case; i.e. for wireless channel $i$, its channel state process $s_{i}(t)$ is modeled by a DTMC with state space $\{0,1\}$ and the transition probability matrix

$$
\mathbf{Q}_{i}:=\left(\begin{array}{cc}
1-p_{i} & p_{i} \\
q_{i} & 1-q_{i}
\end{array}\right)
$$

where $p_{i}$ is the state transition probability of channel $i$ from state 0 to state 1 and $q_{i}$ is the state transition probability of channel $i$ from state 1 to state 0 .

Next, let $\mathbf{S}(t)$ be the channel state process, that is,

$$
\mathbf{S}(t):=\left(s_{1}(t), s_{2}(t), \cdots, s_{N}(t)\right) .
$$

We assume that all $s_{i}(t), 1 \leq i \leq N$ are independent Markov chains, so that $\mathbf{S}(t)$ is also a Markov chain with state space

$$
\mathcal{S}:=\left\{\left(i_{1}, \cdots, i_{N}\right): i_{k}=0 \text { or } 1,1 \leq k \leq N\right\}
$$

and transition probability matrix

$$
\mathbf{R}_{T}:=\mathbf{Q}_{1} \otimes \mathbf{Q}_{2} \otimes \cdots \otimes \mathbf{Q}_{N}
$$

where $\otimes$ denotes the Kronecker product. Note that there are $|\mathcal{S}|=2^{N}$ states in $\mathcal{S}$.

For the channel state process $\mathbf{S}(t)=$ $\left(s_{1}(t), s_{2}(t), \cdots, s_{N}(t)\right)$ at slot $t$, define

$$
N(t):=\sum_{k=1}^{N} s_{k}(t) .
$$

Then, $N(t)$ denotes the number of idle channels at slot $t$.

In what follows we first consider the extreme case where all SUs have full channel state information and analyze the queueing performance of the tagged SU under heterogeneous channels.

As in Section IV we analyze the queueing performance of the tagged SU by first deriving the EBF of the packet service process of the tagged SU. Observing the transition probability matrix of $\mathbf{R}_{T}$ in this case, the EBF of the packet service process of the tagged $\mathrm{SU}$ is given by

$$
\xi_{C}(\theta)=-\frac{\log \delta_{C}(-\theta)}{\theta},
$$

where $\delta_{C}(\theta)$ is the Perron-Frobenius (PF) eigenvalue of the matrix $\boldsymbol{C}(\theta)=\phi(\theta) \mathbf{R}_{T}$, and $\phi(\theta)$ is a diagonal matrix whose element corresponding to state $\left(i_{1}, i_{2}, \cdots, i_{N}\right)$ is given by

$$
E\left[e^{\theta c_{N(t)}(t)} \mid S(t)=\left(i_{1}, i_{2}, \cdots, i_{N}\right)\right] .
$$

Note that (11) does not directly depend on the channel state process $\mathbf{S}(t)$ but directly depend on $N(t)$ because the total number of idle channels determines the service process $c_{N(t)}(t)$ of the tagged SU at slot $t$. Furthermore, from the proof of Theorem 1 we see that the optimal APs are obtained when each diagonal element with $-\theta$, i.e., $E\left[e^{-\theta c_{N(t)}(t)} \mid S(t)=\right.$ $\left.\left(i_{1}, i_{2}, \cdots, i_{N}\right)\right]$ is minimized. Therefore, we obtain the following theorem for heterogeneous wireless channels.

Theorem 4: When all SUs know the channel state process $\mathbf{S}(t)=\left(s_{1}(t), \cdots, s_{N}(t)\right)$, the optimal value of AP, denoted by $a_{\text {full }}^{*}(t)$, at slot $t$ is given by

$$
a_{\text {full }}^{*}(t)=\min \left(\frac{\sum_{k=1}^{N} s_{i}(t)}{M}, 1\right) .
$$


Proof: The theorem immediately follows from the same argument as in the proof of Theorem 1.

When we consider the other extreme case where all SUs have no channel state information, using a similar argument as given above, we can prove the following theorem.

Theorem 5: When all SUs have no information on the channel state process $\mathbf{S}(t)=\left(s_{1}(t), \cdots, s_{N}(t)\right)$, the optimal value of AP, denoted by $a_{n o}^{*}(t)$, at slot $t$ is given by

$$
a_{n o}^{*}(t)=\min \left(\frac{N}{M}, 1\right) \text {. }
$$

\section{NumERICAL RESULTS AND DISCUSSIONS}

In this section we provide some numerical results to validate our analysis and investigate queueing performance behavior. We consider a cognitive radio network with 5 wireless channels and 7 SUs. The state transition probabilities of each wireless channel are given by $p=0.75$ and $q=0.35$ otherwise mentioned. Since the optimal channel access policies of two extreme cases are equivalent when $N \leq M$, we mostly consider the case of full channel state information. We later discuss queueing performances for optimal channel access policies of two extreme cases when $N>M$ in Subsection VII-C.

\section{A. Analysis Validation}

In this subsection, we consider the case of full information on idle channels as previously mentioned.

We first show the validity of our analysis based on the effective bandwidth theory. For this purpose, we use Matlab to simulate the cognitive radio network and obtain the queueing performance of the tagged SU. Two examples are considered: first with APs $\left\{a_{1}, a_{2}, a_{3}, a_{4}, a_{5}\right\}=\{0.8,0.75,0.5,0.45,0.3\}$ and second with the optimal APs $\left\{a_{1}^{*}, a_{2}^{*}, a_{3}^{*}, a_{4}^{*}, a_{5}^{*}\right\}$. The packet arrival process is assumed to be the Poisson process with arrival rate $0.15^{\ddagger}$. The tail probability $P\{q(\infty)>x\}$ is then obtained by averaging over the results from 3 simulation runs, each of which is performed for $10^{5}$ slots. The simulation results when we use the given APs (the optimal APs, resp.) are plotted in Fig. 2(a) (Fig. 2(b), resp.). We also plot the tail probabilities $P\{q(\infty)>x\}$ in (3) for both examples in the figures. As seen in the figures, we see that our analytic results are well matched with simulation results.

We next check the optimality of queueing performance for the optimal APs given in Theorem 1. To demonstrate the validity of Theorem 1 , we vary the value of $a_{3}$ from 0.6 to 0.2 while the other APs $a_{1}, a_{2}, a_{4}$ and $a_{5}$ are invariant and equal to $\left\{a_{1}, a_{2}, a_{4}, a_{5}\right\}=\{0.8,0.75,0.45,0.3\}$. Note that the optimal value $a_{3}^{*}=3 / 7$ by Theorem 1 . The resulting EBFs of the packet service processes and the corresponding tail probabilities $P\{q(\infty)>x\}$ in (3) are plotted in Fig. 3(a) and Fig. 3(b), respectively. To obtain the tail probabilities we assume that the arrival process is the Poisson process with arrival rate 0.15 . As seen in the figures, starting from $a_{3}=0.6$, the EBF of the packet service process increases until $a_{3}$ becomes $a_{3}^{*}=3 / 7$ and then decreases, as do

\footnotetext{
${ }^{\ddagger}$ The unit of the arrival rate is packets/slot in this section.
}

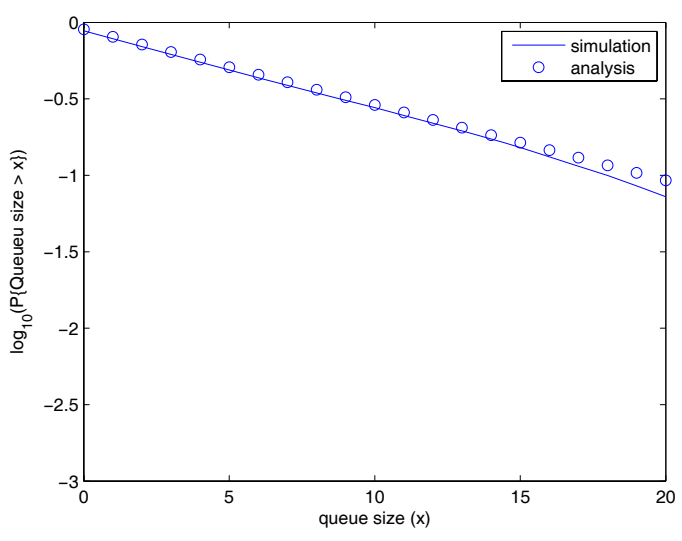

(a) Tail probabilities for the given APs

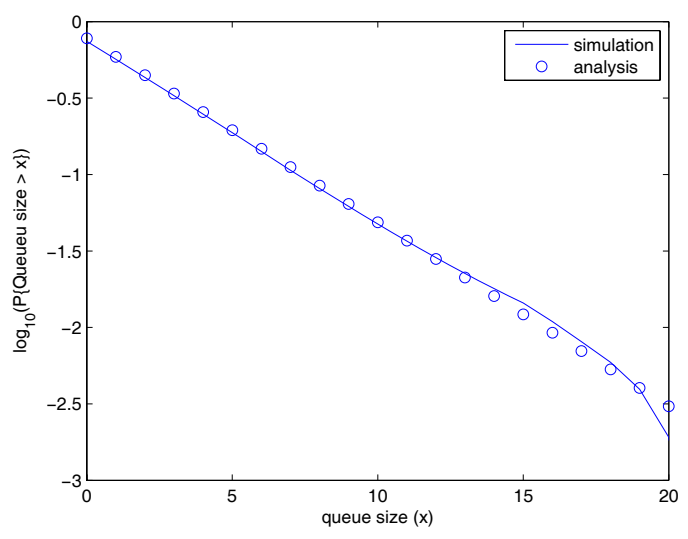

(b) Tail probabilities for the optimal APs

Fig. 2. A comparison of simulation and analytic results.

the corresponding tail probabilities. The same behavior in queueing performance is observed when we change the other APs. As a reference, we also plot the EBF of the packet service process and the corresponding tail probabilities with the optimal APs $\left\{a_{1}^{*}, a_{2}^{*}, a_{3}^{*}, a_{4}^{*}, a_{5}^{*}\right\}$, in the Figs. 3(a), 3(b); we see that the queueing performance is optimized when we use the optimal APs $\left\{a_{1}^{*}, a_{2}^{*}, a_{3}^{*}, a_{4}^{*}, a_{5}^{*}\right\}$ given in Theorem 1 .

The optimality of queueing performance for the optimal AP can be more clearly demonstrated if we consider the case of no channel state information because the case of no channel state information has a single unified AP $a$. When we consider a cognitive radio network with $N=5, M=7$, the optimal AP $a^{*}$ in the case of no information is $5 / 7$. The Poisson arrival process with arrival rate 0.17 is used as the packet arrival process in this case. We plot in Fig. 4 the tail probabilities $P\{q(\infty)>x\}$ in (3) when we change the value of AP $a$ from 0.5 to 0.9 . As seen in the figure, when the optimal value $a^{*}=5 / 7$ is used, the queueing performance becomes optimal, which validates our analytic result in Theorem 2 .

Finally, we validate the relation between optimal channel access policies of two extreme cases as captured in Theorem 3 , via simulation. A Poisson process with arrival rate 0.17 is used as the packet arrival process. As before, the simulation results are obtained by averaging over 3 simulation runs, each with $10^{5}$ slots. The simulation and the analytical results are plotted in Fig. 5, to demonstrate the validity of the analysis 


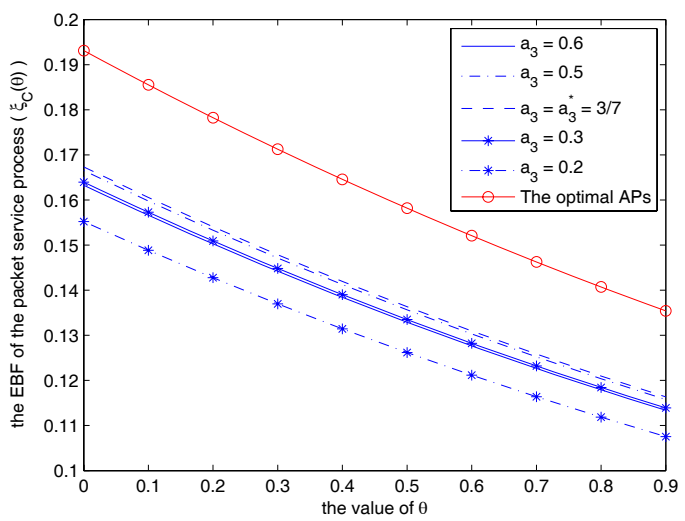

(a) The effective bandwidth function vs. $a_{3}$

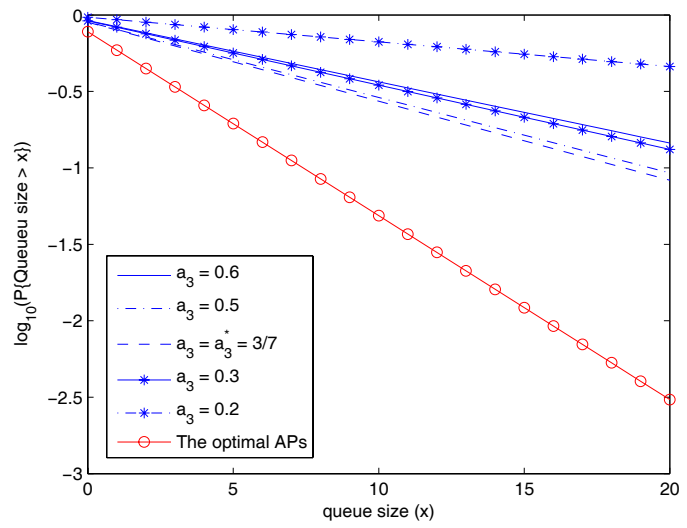

(b) Tail probabilities vs. $a_{3}$

Fig. 3. The queueing performance of channel access policies for the case of full channel state information.

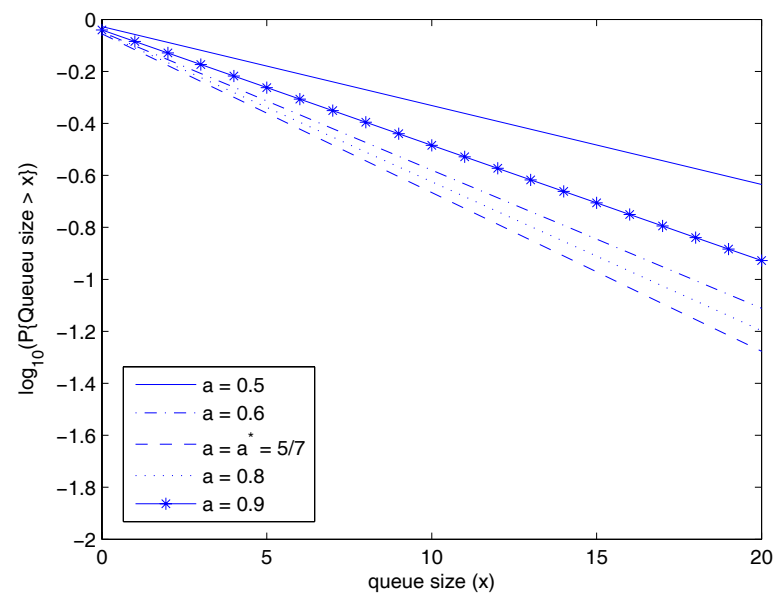

Fig. 4. The queueing performance of channel access policies for the case of no channel state information.

using effective bandwidth theory.

Due to the close correspondence of the simulation and analytic results, we henceforth only use eq. (3) to plot tail probabilities to investigate queueing performance of our optimal channel access policies.

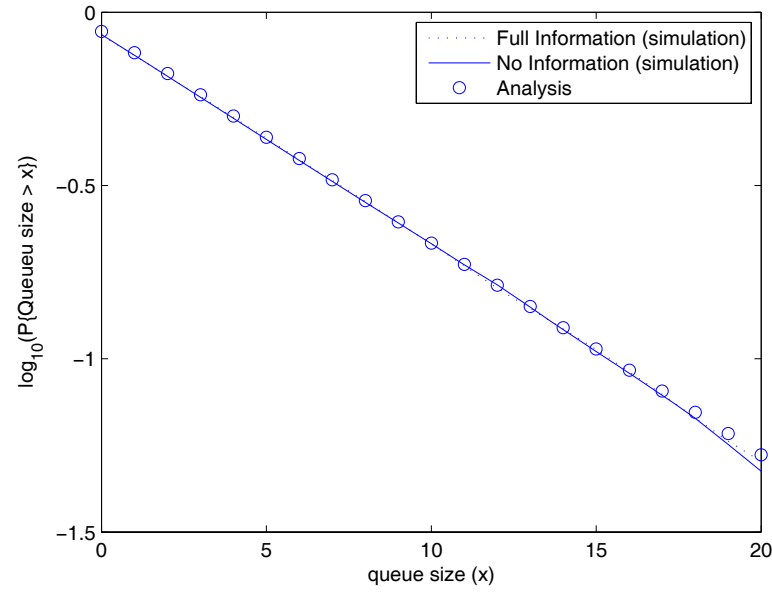

Fig. 5. The equivalence between optimal channel access policies for two extreme cases.

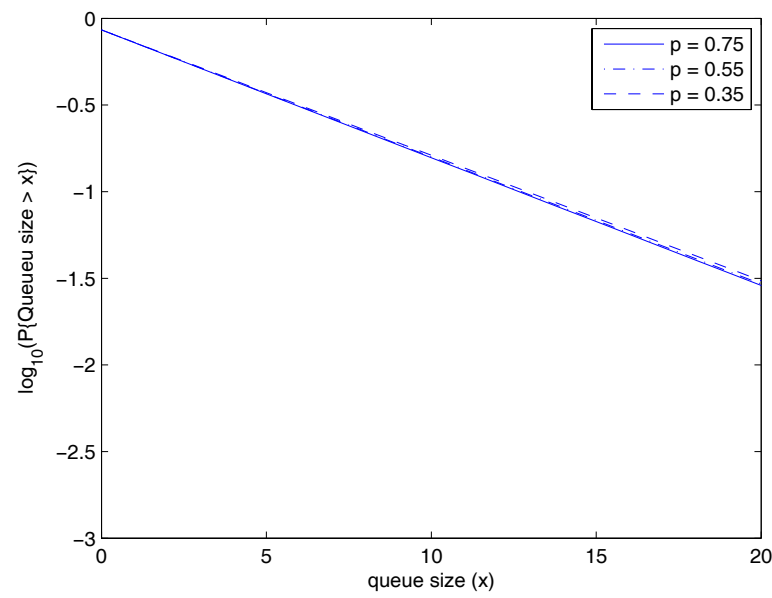

Fig. 6. The queueing performance vs. $p\left(\pi_{1}=0.7, N=5, M=7\right)$.

\section{B. Queuing Performance of The Optimal Channel Access Policy for $N \leq M$}

In this subsection we investigate the effect of the channel state transition probabilities $p$ and $q$ on queueing performance. To this end, we fix the value of $\pi_{1}$, the probability that a wireless channel is idle in the steady state, given in eq. (1). Note that, for given values of $p$ and $\pi_{1}$, the value of $q$ is computed as $q=\frac{1-\pi_{1}}{\pi_{1}} p$. In the numerical study, we use $\pi_{1}=0.7$, and change the value of $p$ from 0.75 to 0.35 . We plot the resulting tail probabilities in Fig. 6 where we use the Poisson process with rate 0.17 as the packet arrival process. Note that both transition probabilities $p$ and $q$ determine the correlation in the wireless channel state process. As seen in the figure, the correlation in the channel state affects the queueing performance even when the value of $\pi_{1}$ (or the stationary probabilities of each wireless channel) is fixed. However, the figure shows that the effect of $p$ and $q$ does not exert a significant effect on queueing performance. This can be explained as follows: in our scheme, SUs observe and occupy idle channels via independent observations and stochastic channel accesses, which decreases the impact of correlation in the underlying channel state process on queueing 


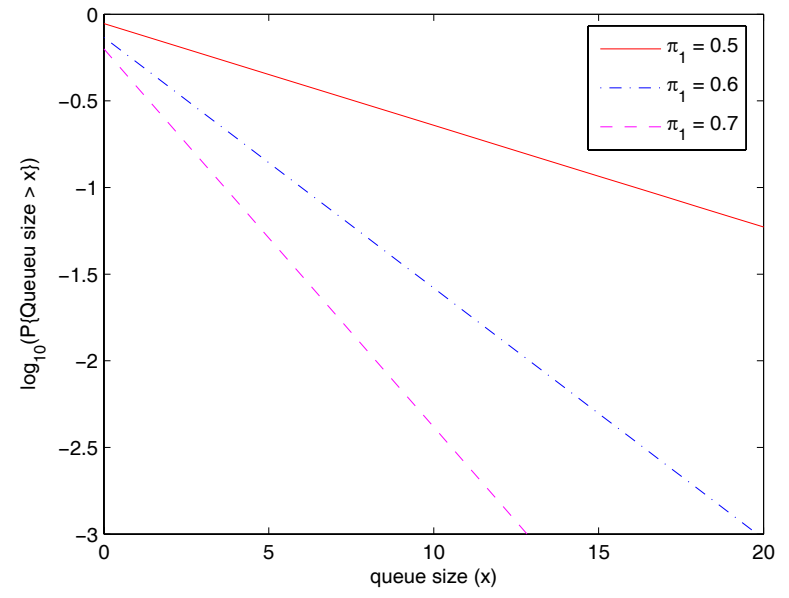

Fig. 7. The queueing performance vs. $\pi_{1}(p=0.75, N=5, M=7)$.

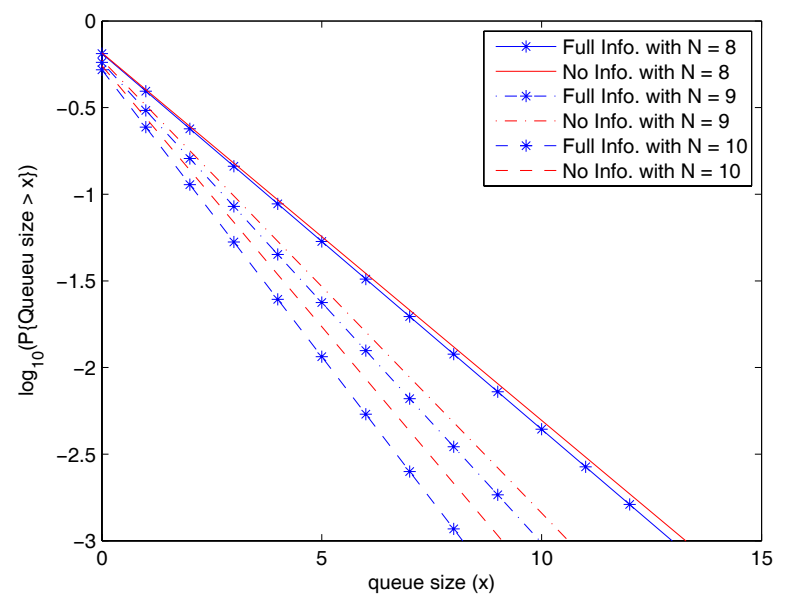

Fig. 8. The queueing performance for $N>M(=7)$.

performance.

To investigate the effect of the stationary probability $\pi_{1}$ on queueing performance, we change the value of $\pi_{1}$ from 0.5 to 0.7 for fixed $p=0.75$. We plot the resulting tail probabilities in Fig. 7 where we use the Poisson process with arrival rate 0.125 as the packet arrival process. As seen in the figure, the value of $\pi_{1}$ affects queueing performance significantly. In addition, with a comparison between Figs. 6 and 7 we conclude that the stationary probability $\pi_{1}$ is more important than individual channel state transition probabilities $p$ and $q$ from the queueing performance viewpoint.

\section{Queuing Performance of The Optimal Channel Access Policy for $N>M$}

We now investigate queueing performances of optimal channel access policies of two extreme cases when $N>M$. For this purpose the Poisson arrival rate is assumed to be 0.2 . We fix $M=7$, change $N$ from 8 to 10 , and plot the resulting tail probabilities in Fig. 8. We see from the figure that, when $N>M$ the knowledge on the channel information is beneficial to SUs; clearly (and as expected), the queueing performance for the case of full information is better than that

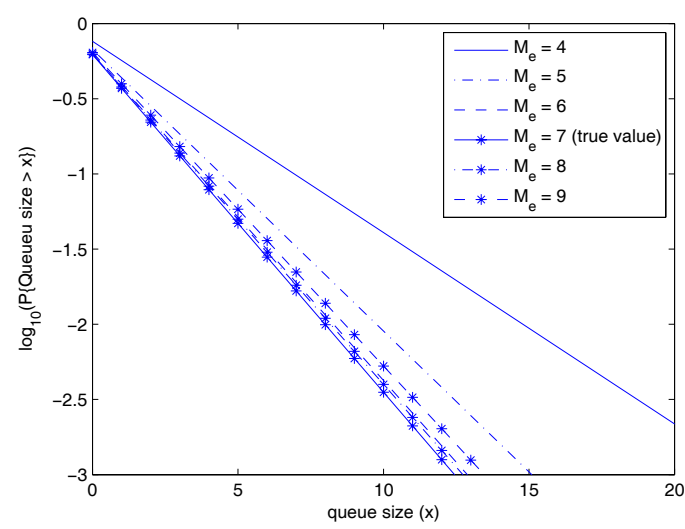

(a) The case of full information

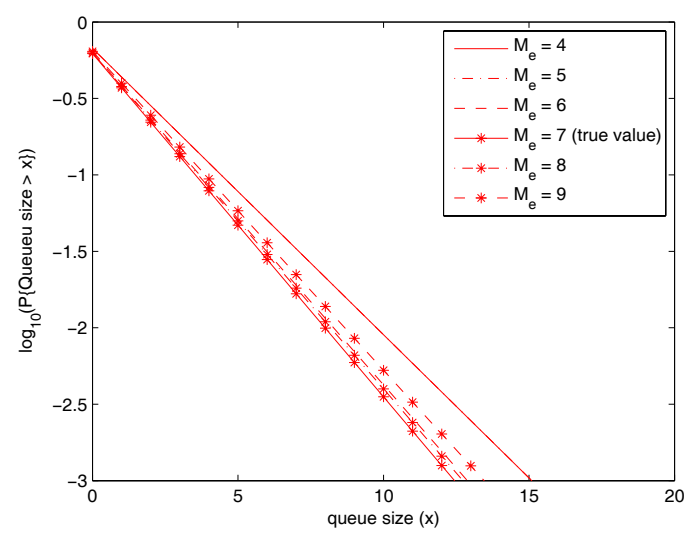

(b) The case of no information

Fig. 9. The sensitivity of the number of SUs $(N=5, M=7)$.

for the case of no information. Furthermore, we see that the difference between queueing performances for two extreme cases becomes more significant as the number of channels increases. Theses results for $N>M$ are quite different from the results for $N \leq M$ and are worth bearing in mind when we design a channel access policy in a cognitive radio network.

\section{Sensitivity to The Number of SUs}

From our analysis, it is evident that (accurate) knowledge on the number of SUs in the cognitive radio network is needed to compute the optimal queueing performance. However, estimation of the number of SUs is not easy in practice. It is hence important to check the sensitivity of queueing performance to the estimation error in the number of SUs in the network. To this end, we assume that there exists an estimation algorithm for the number of SUs in the network, that yields the estimate $M_{e}$. This estimate $M_{e}$ is used to get the optimal APs instead of the true value $M$.

As before, the packet arrival process is Poisson with rate 0.12. The results are plotted in Fig. 9(a) for the case of full channel state information. It is shown in the figure that queueing performance degradation occurs when the tagged SU makes an estimation error in the number $M$ of SUs in the network. In fact, when the value of $M(=7)$ is underestimated such as $M_{e}=4,5$, and 6 , performance degradation is significant. In contrast, when the value of $M$ is overestimated 


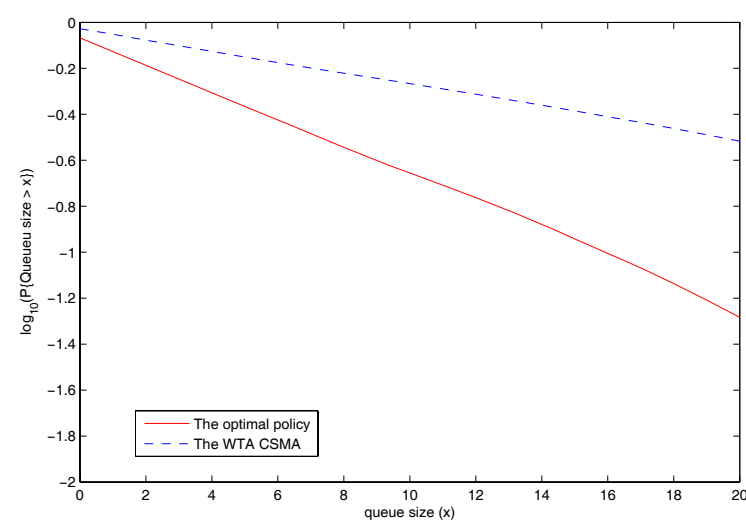

Fig. 10. Performance comparison.

such as $M_{e}=8$ and 9 , performance degradation is relatively not significant.

We next consider the case of no information on idle channels. Here we use the same packet arrival process, i.e., Poisson with rate 0.12. The results in Fig. 9(b) show similar results to the case of full channel state information. However, by comparing Figs. 9(a) and 9(b), we see that the impact of estimation error is more significant in the case of full information than in the case of no information. Hence, even though the optimal channel access policies with full and no information on idle channels are identical as given in Theorem 3 , their practical performance could be different due to the different sensitivities in the estimation of $M$.

\section{E. Performance Comparison}

We compare our optimal channel access policy with the channel access policy based on the $r$-persistent CSMA, e.g., [4]. From queuing performance perspective, the main difference between these two policies is that in the latter, each SU contends for idle channels at each slot; the winner uses all idle channels to transmits its packets as in [4]. In contrast, each SU randomly selects one of the available idle channels in our policy. Due to the above, we call the latter policy the WTA (Winner Takes All) CSMA.

The WTA CSMA considered here is given as follows. There are $N$ channels and $M$ SUs in the network. Each SU uses the $r$-persistent CSMA to reserve idle channels at each slot. For this, each SU transmits a reservation packet with probability $r$ at each slot, independently of all other SUs. If there are two or more SUs simultaneously transmitting reservation packets at a slot, then all reservation packets collide and consequently no SUs can reserve idle channels. On the other hand, if there is only one $\mathrm{SU}$ transmitting a reservation packet at a slot, then the SU becomes the winner and reserves all idle channels at the slot. If there are $k$ idle channels at the slot, the winning SU transmits $k$ packets in the WTA CSMA.

Assuming that all untagged SUs are saturated as in Section $\mathrm{V}$, the probability that the tagged $\mathrm{SU}$ is the winner in the WTA CSMA equals $r(1-r)^{M-1}$. To optimize the queueing performance, this probability should be maximized, which occurs when $r^{*}=1 / M$.
We use $r^{*}=1 / M$ and simulate the WTS CSMA when $N=5$ and $M=7$. For the tagged SU, the Poisson arrival process with arrival rate 0.17 is used in the simulation. We also simulated our optimal channel access policy for the case of no channel state information. The results are plotted in Fig. 10. Interestingly, our optimal channel access policy outperforms the WTA CSMA. This implies that the queueing performance can be improved when the idle channels are distributed among multiple SUs as in our channel access policy.

\section{F. Relation of Our Access Policy to IEEE 802.11 DCF}

We close this paper with a discussion on how our optimal channel access policy is related to the IEEE 802.11 Distributed Coordination Function (DCF). In the IEEE 802.11 DCF, each user having a packet to transmit, senses channel state continually for a DIFS interval and uses an exponential backoff scheme to access the wireless channel [27]. Each user waits until its backoff timer value counts down to 0 before it transmits. The transmission frequency of each user (analogous to the probability that a user transmits in a slot in our model) is denoted by $\tau$ in [27]. It is known that the probability $\tau$ should be set ${ }^{\S}$ to be inversely proportional to the number of users in the network to achieve maximum network throughput.

Our channel access policy shares similar features with DCF in the following sense: each $\mathrm{SU}$ has to wait to transmit a packet, by first becoming active, which is stochastically controlled by the APs. The APs in our policy corresponds to the access probability $\tau$ in Bianchi's model for DCF. Our results exhibit the same dependance, i.e. the optimal APs is inversely proportional to $M$, the number of contending SUs, for the two information regimes assumed. Further, our result is a generalization of Bianchi's in the sense that we consider availability of multiple (idle) channels simultaneously; as expected, the optimal APs are proportional to $k$, the number of available (idle) channels.

\section{CONCLUSIONS}

In this paper, we considered a cognitive radio network where multiple secondary users contend to access wireless channels. We proposed a new channel access policy where each secondary user stochastically determine whether to access a wireless channel based on a given access probability.

In this paper we considered two extreme cases - the case of full information on idle channels and the case of no information on idle channels. To obtain the optimal access probabilities for two extreme cases, we analyzed the queueing performance of an arbitrary secondary user with the help of the effective bandwidth theory. Throughout our analysis we showed how to design the optimal channel access policies for two extreme cases and an equivalent relation between the optimal channel access policies of two extreme cases. We analyzed a homogeneous channel model, but later extended our analysis to a heterogeneous channel model. Our numerical results showed that our analytic results are valid. In addition, we investigated the effects of system parameters such as channel state transition probabilities, the number of channels, and

\footnotetext{
$\S$ The value of $\tau$ is set indirectly, by adjusting the backoff window size.
} 
the number of secondary users on the queueing performance of an arbitrary secondary user.

\section{ACKNOWLEDGMENTS}

The authors would like to thank the anonymous reviewers for their valuable comments and suggestions, which improved the presentation of this paper. This research was supported by Basic Science Research Program through the National Research Foundation of Korea (NRF) funded by the Ministry of Education, Science and Technology (No. 2011-0022026).

\section{REFERENCES}

[1] Federal Comunications Commission, "Spectrum Policy Task Force," Rep. ET Docket No. 02-135, Nov. 2002.

[2] Federal Comunications Commission, "Notice of proposed rule making and order," Rep. ET Docket N0. 03-222, Dec. 2003.

[3] M. McHenry, "Spectrum white space measurements," 2003 New America Foundation BroadBand Forum.

[4] H. Su and X. Zhang, "Cross-layer based opportunistic MAC protocols for QoS provisionings over cognitive radio wireless networks," IEEE J. Sel. Areas Commun., vol. 26, no. 1, pp. 118-129, Jan. 2008.

[5] Q. Zhao, B. Krishnamachari, and K. Liu, "On myopic sensing for multichannel opportunistic access: structure, optimality, and performance," IEEE Trans. Wireless Commun., vol. 7, no. 12, pp. 5431-5440, Dec. 2008.

[6] C.-S. Chang, Performance Guarantees in Communication Networks. Springer-Verlag, 2000.

[7] D. Wu and R. Negi, "Effective capacity: a wireless link model for support of quality of service," IEEE Trans. Wireless Commun., vol. 2, no. 4, pp. 630-643, July 2003.

[8] F. Ishizaki and G. U. Hwang, "Cross-layer design and analysis for wireless networks using the effective bandwidth function," IEEE Trans. Wireless Commun., vol. 6, no. 9, pp. 3214-3219, Sep. 2007.

[9] X. Zhang and Q. Du, "Cross-layer modeling for QoS-driven multimedia multicast/broadcast over fading channels in mobile wireless networks," IEEE Commun. Mag., vol. 45, no. 8, pp. 62-70, Aug. 2007.

[10] H. Kim and K. G. Shin, "Efficient discovery of spectrum opportunities with MAC-layer sensing in cognitive radio networks," IEEE Trans. Mobile Comput., vol. 7, pp. 533-545, May 2008.

[11] C. Cormio and K. R. Chowdhury, "A survey on MAC protocols for cognitive radio networks," Ad Hoc Networks, vol. 7, pp. 1315-1329, 2009.

[12] A. Ghasemi and E. S. Sousa, "Optimization of spectrum sensing for opportunistic spectrum access in cognitive radio networks," in Proc. 2007 IEEE Consumer Commun. Networking Conf., pp. 1022-1026.

[13] P. Wang, L. Xiao, S. Zhou, and J. Wang, "Optimization of detection time for channel efficiency in cognitive radio systems," in Proc. 2008 IEEE Wireless Commun. Networking Conf., pp. 111-115.

[14] W. Y. Lee and I. F. Akyildiz, "Optimal spectrum sensing framework for cognitive radio networks," IEEE Trans. Wireless Commun., vol. 7, no. 10, pp. 3845-3857, Oct. 2008.

[15] J. Jia, Q. Zhang, and X. Shen, "HC-MAC: a hardware-constrained cognitive MAC for efficient spectrum management," IEEE J. Sel. Areas Commun., vol. 26, no. 1, Jan. 2008.

[16] L. Ma, X. Han, and C. C. Shen, "Dynamic open spectrum sharing for wireless ad hoc networks," in Proc. 2007 IEEE DySPAN, pp. 547-558.

[17] C. Cordeiro and K. Challapali, "C-MAC: a cognitive MAC protocol for multichannel wireless networks," in 2007 Proc. IEEE DySPAN, pp. 147-157.

[18] Q. Zhao, L. Tong, A. Swami, and Y. Chen, "Decentralized cognitive MAC for opportunistic spectrum access in ad hoc networks: a POMDP framework," IEEE J. Sel. Areas Commun., vol. 25, no. 3, pp. 589-600, 2007.

[19] S. Shankar, "Squeezing the most out of cognitive radio: a joint MAC/PHY perspective," in Proc. 2007 IEEE ICASSP, pp. IV-1361IV-1364.

[20] L. C. Wang, Y. C. Lu, C. W. Wang, and D. S. L. Wei, "Latency analysis for dynamic spectrum access in cognitive radio: dedicated or embedded control channel?" in Proc. 2007 IEEE PIMRC.

[21] M. M. Rashid, Md. J. Hossain, E. Hossain, and V. K. Bhargave, "Opportunistic spectrum scheduling for multiuser cognitive radio: a queueing analysis," IEEE Trans. Wireless Commun., vol. 8, no. 10, Oct. 2009.
[22] A. Laourine, S. Chen, and L. Tong, "Queueing analysis in multichannel cognitive spectrum access: a large deviation approach," in Proc. 2010 IEEE INFOCOM.

[23] S. Wang, J. Zhang, and L. Tong, "Delay analysis for cognitive radio networks with random access: a fluid queue view," in Proc. 2010 IEEE INFOCOM.

[24] C.-S. Chang and J. A. Thomas, "Effective bandwidths in high-speed digital networks," IEEE J. Sel. Areas Commun., vol. 3, no. 6, pp. 10911100, Aug. 1995.

[25] B. L. Mark and G. Ramamurthy, "Real-time estimation and dynamic renegotiation of UPC parameters for arbitrary traffic sources in ATM networks," IEEE/ACM Trans. Netw., vol. 6, no. 6, pp. 811-827, Dec. 1998.

[26] H. Minc, Nonnegative Matrices. John Wiley \& Sons, Inc., 1988

[27] G. Bianchi, "Performance analysis of the IEEE 802.11 distributed coordination function," IEEE J. Sel. Areas Commun., vol. 18, no. 3, pp. 535-547, Mar. 2000.

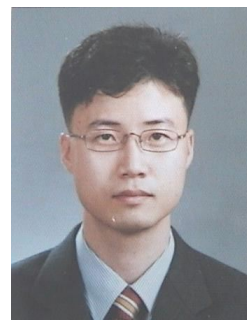

Gang Uk Hwang (M'03) received his B.Sc., M.Sc., and Ph.D. degrees in mathematics (applied probability) from Korea Advanced Institute of Science and Technology (KAIST), Daejeon, Republic of Korea, in 1991, 1993 and 1997, respectively. From February 1997 to March 2000, he was with the Electronics and Telecommunications Research Institute (ETRI), Daejeon, Republic of Korea. From March 2000 to February 2002, he was a visiting scholar at the School of Interdisciplinary Computing and Engineering at the University of Missouri - Kansas City. Since March 2002, he has been with the Department of Mathematical Sciences and Telecommunication Engineering Program at KAIST, where he is currently an Associate Professor. During a sabbatical leave from August 2010 to July 2011, he was a visiting scholar at the Department of Electrical Engineering, University of Washington, Seattle, where he worked on performance modeling and analysis of cognitive radio networks. His research interests include teletraffic theory, performance analysis of communication systems, quality of service provisioning for wired/wireless networks and cross-layer design, and optimization for wireless networks.

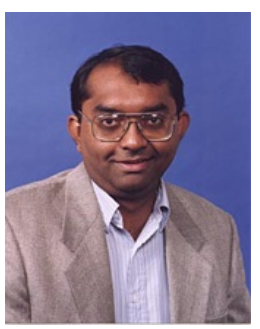

Sumit Roy (LF'07) received the B.Tech. degree from the Indian Institute of Technology (Kanpur) in 1983, and the M.S. and Ph.D. degrees from the University of California (Santa Barbara), all in electrical engineering in 1985 and 1988, respectively, as well as an M.A. in statistics and applied probability in 1988 . His previous academic appointments were at the Moore School of Electrical Engineering, University of Pennsylvania, and at the University of Texas, San Antonio; presently, he is a Professor of electrical engineering at the University of Washington, where his research interests include the analysis/design of communication systems/networks, with an emphasis on next generation mobile and wireless networks. He spent 2001-2003 on academic leave at the Intel Wireless Technology Lab as a Senior Researcher engaged in research and standards development for ultra-wideband systems (Wireless PANs) and next generation high-speed wireless LANs. He has over 60 archival journal and 90 conference publications and his research has been consistently funded by various United States national agencies. He was elevated to IEEE Fellow by the IEEE Communications Society in 2007 for his "contributions to multiuser communications theory and cross-layer design of wireless networking standards."

His activities for the IEEE Communications Society include membership of several technical and conference program committees (notably Technical Committee on Cognitive Networks). He has served as an Associate Editor for all the major ComSoc publications in his area at various times, including the IEEE TRANSACTIONS ON COMMUNICATIONS and the IEEE TRANSACTIONS ON WIRELESS COMMUNICATIONS. He currently serves on the Editorial Board for the IEEE TRANSACTIONS ON COMMUNICATIONS, IEEE JOURNAL ON INTELLIGENT TRANSPORTATION SYSTEMS, and the new IEEE TRANSACTIONS ON SMART GRIDS. His other notable professional activities include reviewing proposals for various international research panels (Singapore, HongKong, Qatar, etc.) and serving as an external examiner for Ph.D. theses (Singapore, Australia, Canada, etc.). 